\title{
Non-uniform nanosecond gate-delay of hybrid pixel detectors
}

\section{Roman Shayduk, David Pennicard, Konstantin Krausert, Peter Gaal, Sergey Volkov, Vedran Vonk, Uta Hejral, Maciej Jankowski, Matthias Reinhardt, Wolfram Leitenberger and Andreas Stierle}

J. Synchrotron Rad. (2017). 24, 1082-1085

\section{IUCr Journals CRYSTALLOGRAPHY JOURNALS ONLINE}

Copyright (C) International Union of Crystallography

Author(s) of this paper may load this reprint on their own web site or institutional repository provided that this cover page is retained. Republication of this article or its storage in electronic databases other than as specified above is not permitted without prior permission in writing from the IUCr.

For further information see http://journals.iucr.org/services/authorrights.html 




ISSN 1600-5775

Received 15 February 2017

Accepted 19 June 2017

Edited by G. Grübel, HASYLAB at DESY, Germany

Keywords: pump-probe X-ray diffraction; hybrid pixel $\mathrm{X}$-ray detector; triggering; timing; jitter.

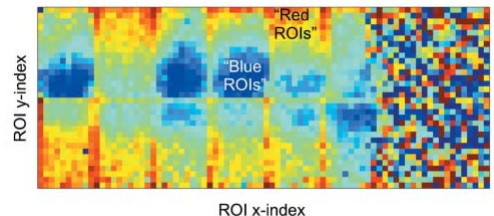

C 2017 International Union of Crystallography

\section{Non-uniform nanosecond gate-delay of hybrid pixel detectors}

\author{
Roman Shayduk, ${ }^{\text {a* }}$ David Pennicard, ${ }^{\text {a }}$ Konstantin Krausert, ${ }^{\text {a }}$ Peter Gaal, ${ }^{\mathrm{b}}$ \\ Sergey Volkov, ${ }^{\text {a }}$ Vedran Vonk, ${ }^{\mathrm{a}}$ Uta Hejral, ${ }^{\mathrm{a}}$ Maciej Jankowski, ${ }^{\mathrm{c}}$ \\ Matthias Reinhardt, ${ }^{d}$ Wolfram Leitenberger ${ }^{d}$ and Andreas Stierle ${ }^{a, b}$
}

\begin{abstract}
${ }^{\mathbf{a}}$ Deutsches Elektronen-Synchrotron (DESY), D-22603 Hamburg, Germany, ${ }^{\mathbf{b}}$ Physics Department, University of Hamburg, Germany, ${ }^{\mathbf{c} E u r o p e a n}$ Synchrotron Radiation Facility (ESRF), Grenoble, France, and ${ }^{\mathbf{d}}$ Helmholtz-Zentrum Berlin für Materialien und Energie GmbH, Wilhelm-Conrad-Röntgen Campus, BESSY II, Albert-Einstein-Strasse 15, 12489 Berlin, Germany.*Correspondence e-mail: roman.shayduk@desy.de
\end{abstract}

A simple experiment to characterize the gating properties of X-ray area detectors using pulsed X-ray sources is presented. For a number of timeresolved experiments the gating uniformity of area detectors is important. Relative gating delays between individual modules and readout chips of PILATUS2 series area X-ray detectors have been observed. For three modules of a PILATUS $300 \mathrm{~K}-\mathrm{W}$ unit the maximum gating offset between the modules is found to be as large as $30 \mathrm{~ns}$. On average, the first photosensor module is found to be triggered $15 \mathrm{~ns}$ and $30 \mathrm{~ns}$ later than the second and the third modules, respectively.

\section{Introduction}

Synchrotron storage rings generate $\mathrm{X}$-ray radiation that consists of a series of short X-ray photon bursts separated by, essentially, photon-free gaps. Many storage rings are operated in the so-called hybrid and/or single-bunch mode providing an $\mathrm{X}$-ray pulse obtained from an isolated electron bucket that is separated from the rest of the bunches by two electron-free gaps hundreds of nanoseconds long. It has been shown that these isolated pulses can be gated by modern area X-ray detectors (Ejdrup et al., 2009; Reinhardt et al., 2016). Here all the unwanted signal is masked and it makes it possible to carry out synchrotron-based pump-probe time-resolved X-ray scattering experiments with time resolution down to hundreds of femtoseconds (Schoenlein et al., 2000) without the need of a mechanical chopper (Wulff et al., 2003).

Although hybrid pixel area X-ray detectors use a large-area single-crystal photosensor, the spatial resolution is achieved via hybrid pixel architecture (Broennimann et al., 2006; Dinapoli et al., 2011; Ponchut et al., 2002). The readout electronics constituting a photosensor are usually modular. When such a detector receives a gate signal it is important that the signal is passed to and processed by all the readout modules simultaneously.

In this short communication we describe a simple experiment that is aimed at characterizing the area X-ray detector triggering uniformity. In particular we report on the gating non-uniformity of two area X-ray detectors PILATUS 300$\mathrm{K}-\mathrm{W}$ and PILATUS 100K employed in real synchrotron-based pump-probe X-ray diffraction experiments. The carried-out 'pulsed flat intensity field' experiments could serve as a standard tool to characterize the gating uniformity of a whole class of hybrid pixel area X-ray detectors. 
(a)
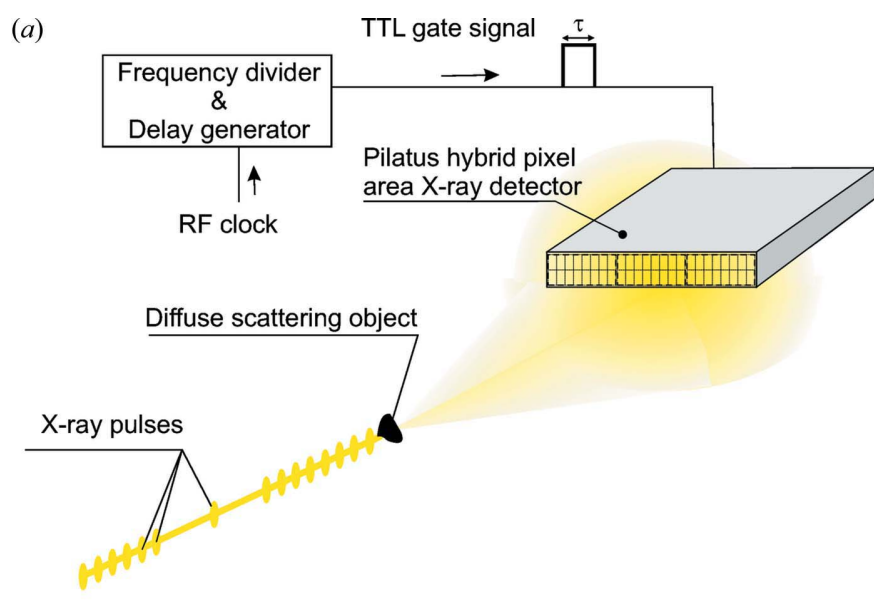

(b)

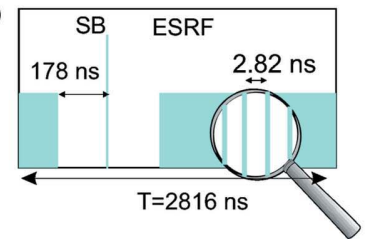

Figure 1

(a) A simple sketch of the experimental setup. (b) Schemes of bunch structures at ESRF and at BESSY storage rings.

\section{Experimental setups}

\subsection{ESRF setup}

The experiment with the PILATUS $300 \mathrm{~K}-\mathrm{W}$ unit was carried out at the ID03 surface X-ray diffraction beamline at the European Synchrotron Radiation Facility (ESRF). The storage ring was operated in hybrid 7/8 bunch-filling mode with 868 electron bunches filled with $0.23 \mathrm{~mA}$ per bunch and one isolated single $1 \mathrm{~mA}$ bunch placed in the center of a 356 ns-wide electron bunch-free gap as schematically shown in Fig. 1(b). We used monochromatic X-rays with an energy of $15 \mathrm{keV}$. The direct beam intensity was of the order of $10^{12}$ photons s $^{-1}$.

Fig. 1(a) shows a sketch of the experiment. The X-ray detector was placed in the region of strong diffuse scattering in the vicinity of the non-attenuated direct X-ray beam. The detector was operated in the so-called 'external enable' triggering $\operatorname{mode}^{\mathbf{1}}$ and received a TTL gate signal from a DG645 delay generator (Stanford Inc.). The signal had sharp rising and falling edges of around $200 \mathrm{ps}$ and a width of $50 \mathrm{~ns}$. In the experiment, the DG645 signals were phase-locked to the storage-ring revolution frequency and the repetition rate of the signals were frequency divided to reach a rate of $\sim 1 \mathrm{kHz}$. The reduction of the repetition rate to $1 \mathrm{kHz}$ was required by the laser used in the ESRF experiment.

\subsection{BESSY setup}

The experiment with the PILATUS $100 \mathrm{~K}$ single-module unit was carried out at the KMC3 beamline of the BESSY II storage ring in Berlin, Germany. During the experiment,

\footnotetext{
${ }^{1}$ As described in the PILATUS manual.
}

BESSY II was operated in the so-called 'low- $\alpha$ multi-bunch hybrid mode'. In this operation mode an isolated electron bunch is placed near the center of a 184 ns-wide bunch-free gap as schematically shown in Fig. 1(b). As in the ESRF experiment the detector was operated in the 'external enable' triggering mode and received a phase-locked 20 ns-long TTL gate signal. The repetition rate of the signal in this experiment was set to $1.25 \mathrm{MHz}$, equal to the BESSY revolution frequency. The X-ray energy used was $11.9 \mathrm{keV}$.

In practice, one readout frame of a PILATUS detector in the 'external enable' gated mode synthesizes a digital image that stores photon hits in each pixel due to multiple ultrashort gated exposures. For example, for a $1 \mathrm{kHz}$ trigger rate a $1 \mathrm{~s}$ 'exposure' produces one digital image that is the sum of 1000 gated exposures. In the 'external enable' triggered mode it was possible to trigger the detector exposure at a repetition rate that exceeds by far the chip readout-limited rate of around $300 \mathrm{~Hz}$. The internal mechanism of a single ultrashort gate exposure controlled by an external gate is a part of the PILATUS detector architecture (Kraft et al., 2009) and will not be discussed in this article.

\section{Results}

\subsection{Test of PILATUS $300 \mathrm{~K}-\mathrm{W}$}

During the experiment the detector was kept at a fixed position. The readout period in the experiments was $1 \mathrm{~s}$, equivalent to 1000 exposures at $1 \mathrm{kHz}$. The starting point of the gate signal with respect to the single bunch was changed in steps of $1 \mathrm{~ns}$ over the revolution period of $2.8 \mu \mathrm{s}$. The integrated counts of different regions of interest (ROIs) of the photosensor were recorded as a function of the gate delay. Fig. 2(b) shows two overlayed histograms of integrated counts as a function of gate delay taken from two different ROIs. To compare how different regions of a detector respond to the gate signal, ROI $(1,1)$ is placed near the upper left corner of the detector and $\operatorname{ROI}(14,2)$ is the region in the center of the detector as indicated in Fig. 2(b).

From the data we unambiguously determine at which gate delay only those X-ray photons are counted that correspond to the isolated electron bunch i.e. 'single bunch' (SB) X-ray photons. The 'zero point' of the gate delay in the plot is chosen at the single-bunch center-of-mass (COM) of ROI $(1,1)$. These photons produce count events that form the trapezoidal distribution around zero. One can also see the $~ 90$ ns-long counts-free regions corresponding to the two $178 \mathrm{~ns}-$ long bunch-free time gaps of the 7/8+1 ESRF filling mode (one bunch placed in the center of the 356 ns-long bunch-free gap). The higher counting statistics on the left and on the right sides of the histograms are due to the capture of X-ray photons generated by 2.8 ns-separated electron bunches of ESRF. Due to the detector response time limitations, the multi-bunch time structure is not resolved in this experiment.

One striking feature of this particular PILATUS 300K-W module is the $30 \mathrm{~ns}$ offset of the COM of ROI(1,1) counts compared with the ROI $(14,2)$ counts COM. In addition, the 
SB counts falling edge of $\operatorname{ROI}(14,2)$ is observed about $20 \mathrm{~ns}$ before the SB counts falling edge of ROI(1,1). The pulse length of a single X-ray pulse is of the order of $100 \mathrm{ps}$ and the spatial distribution of the synchrotron radiation flux over the detector photosensor is rather homogeneous in this experiment. Therefore, these systematic time offsets in the histograms which depend on the position of the photosensor area reflect the gating property of the detector readout electronics. It is unlikely that the observed time offsets of 20-30 ns stem from signal propagation in the detector. This would require a cable length of $4-6 \mathrm{~m}$ in the detector. We exclude any environmental effects as the X-ray hutch is equipped with air-conditioning that supports constant temperature and humidity. Furthermore, the environment or the detector setting fluctuations during the experiment would most likely affect the absolute delays. The observed statistically averaged relative delays as a function of photosensor region would stay intact.

Figs. 2(b) and 2(c) show color-coded maps of the timings of a SB's rising and falling edges for the whole area of the PILATUS 300K detector for all 48 different readout chips of the detector. The rising and the falling edges were determined by fitting Gaussians to the differentiated data of Fig. 2(a) type. The standard deviations of the fits were of the order of $3.8 \mathrm{~ns}$. These data clearly show differences in the measured SB position depending on the module. The maximum gating difference for this PILATUS 300K-W unit was of the order of $30 \mathrm{~ns}$ as determined in this experiment and was between the


Figure 2

(a) Count-rate histograms as a function of gate delay for two different ROIs. (b) PILATUS $300 \mathrm{~K}-\mathrm{W}$ photosensor area divided on a grid of $24 \times 2$ ROIs. The color code corresponds to the position of the singlebunch histogram left shoulder. (c) Same as in (b) but the color code corresponds to the position of the single-bunch histogram right shoulder. (d) Detector image of integrated photon counts after $10^{7}$ gated exposures taken at zero relative gate delay. The data are taken from a real experiment. first and the third modules. It is not yet clear whether this offset is peculiar to this particular detector or whether it is a typical property of the PILATUS series of multi-module detectors. Within each module, the variation in timing behavior is significantly smaller (less than $12 \mathrm{~ns}$ ) and forms a fairly random pattern. On average, the first photosensor module is triggered $15 \mathrm{~ns}$ and $30 \mathrm{~ns}$ later than the second and the third modules, respectively.

In Fig. 2(d) one can see an example of a gated PILATUS $300 \mathrm{~K}-\mathrm{W}$ image taken in a real pump-probe surface X-ray diffraction experiment. The image is taken at zero relative delay [see Fig. 2(a)]. The complex intensity structure in the PILATUS image is essentially coming from the X-ray powder diffraction rings of the Be chamber used in the experiment and from the diffuse scattering that is shadowed by the detector flight tube. However, on top of the complex intensity structure one clearly distinguishes rectangular regions of 'dimmed' count rates. These regions reveal the $2 \times 8$ readout chip structure of a single module. The 'dimmed chip' effect nearly disappears when the relative gate delay is set to $-10 \mathrm{~ns}$ and the gate width is extended to $100 \mathrm{~ns}$. For the 50 ns-long gate the chip contrast was clearly present in detector images for all gate delays. Hence, using the 50 ns-long gate the simultaneous gating of all the three modules was not possible.

\subsection{Test of PILATUS $100 \mathrm{~K}$}

Fig. 3 represents the data similar to those shown in Figs. 2(a)-2(c) but acquired for a different single-module PILATUS $100 \mathrm{~K}$ area X-ray detector at the BESSY synchrotron. In this experiment the data were taken with much higher statistics. Fig. 3(a) shows the number of counted photons as a
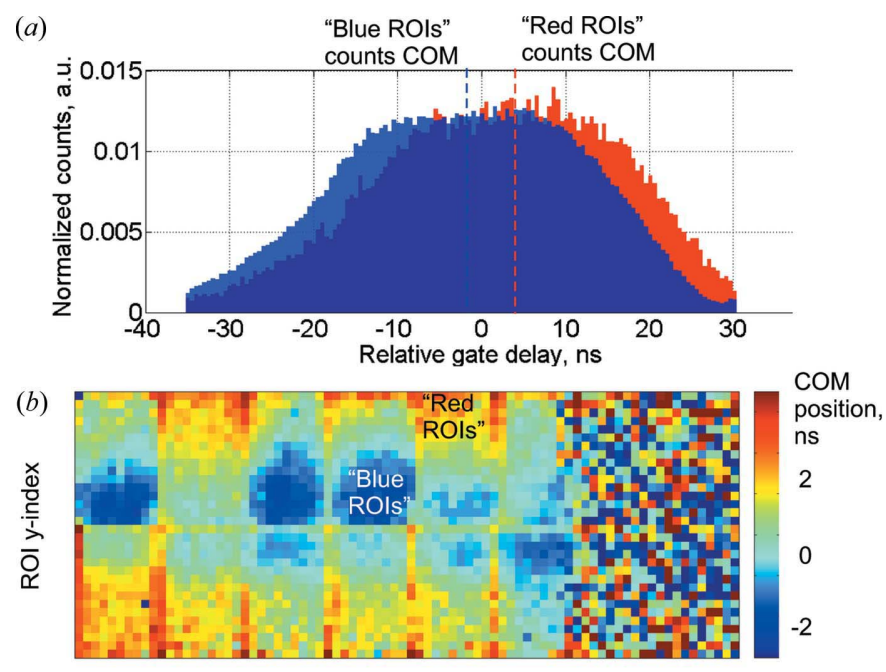

ROI x-index

Figure 3

(a) Two overlayed count-rate histograms as a function of gate delay for the 'blue' and the 'red' ROIs as indicated in $(b)$. Dashed vertical lines indicate the 'center of mass' (COM) position of the corresponding histogram. (b) PILATUS 100K photosensor area divided on a grid of $82 \times 32$ ROIs. The color code corresponds to the COM position of the single-bunch histogram. The statistical noise seen on the right-hand side of the photosensor area is due to the much lower X-ray photon hit rate in this region. 
function of gate delay near the SB for two different ROIs. Here we used ROIs of $6 \times 6$ PILATUS pixels. Such ROIs were distributed on a grid to cover the entire PILATUS $100 \mathrm{~K}$ photosensor. In Fig. 3(b) we plot a color map of SB COM position as a function of ROI on the grid. By doing so, the $2 \times 8$ readout chip structure of the PILATUS $100 \mathrm{~K}$ detector module becomes visible. Although the apparent delays are quite small now, some chips still represent a systematic gate offset of up to $5 \mathrm{~ns}$ on average. There is also an apparent COM position gradient within each chip observed. The standard deviation of the COM determination in this experiment is limited only by the counting statistics and is of the order of 200 ps.

\section{Conclusion}

We describe a simple experiment to characterize the gating properties of X-ray area detectors using pulsed X-ray sources.

The gating performance of two PILATUS 300K-W and PILATUS $100 \mathrm{~K}$ detectors employed in real synchrotronbased pump-probe X-ray diffraction experiments is discussed. Systematic gating offsets of up to $30 \mathrm{~ns}$ between three modules constituting the PILATUS $300 \mathrm{~K}-\mathrm{W}$ photosensitive area were observed. On average, the second and the third photosensor modules were found to be triggered $15 \mathrm{~ns}$ and $30 \mathrm{~ns}$ later than the first module, respectively. Based on the results one can speculate that the gate delay would grow progressively with the number of modules used, i.e. for a PILATUS 6M it could be as large as $300 \mathrm{~ns}$. The results of our experiments on a PILATUS $100 \mathrm{~K}$ detector are in agreement with earlier work (Ejdrup et al., 2009), demonstrating that the detector can be used to achieve X-ray pulse duration-limited time resolution in synchrotron-based pump-probe experiments. However, the multi-modular unit PILATUS $300 \mathrm{~K}-\mathrm{W}$ requires the analysis of gating performance in multiple regions of the photosensitive area to determine the optimal gate timing in a pump-probe experiment.

The experiment described in this article is proposed as the standard evaluation procedure for hybrid pixel area X-ray detectors gating capabilities. The results of these experiments deliver important information required for the proper implementation of area X-ray detectors in synchrotron-based pump-probe experiments.

\section{References}

Broennimann, Ch., Eikenberry, E. F., Henrich, B., Horisberger, R., Huelsen, G., Pohl, E., Schmitt, B., Schulze-Briese, C., Suzuki, M., Tomizaki, T., Toyokawa, H. \& Wagner, A. (2006). J. Synchrotron Rad. 13, 120-130.

Dinapoli, R., Bergamaschi, A., Henrich, B., Horisberger, R., Johnson, I., Mozzanica, A., Schmid, E., Schmitt, B., Schreiber, A., Shi, X. T. \& Theidel, G. (2011). Nucl. Instrum. Methods Phys. Res. A, 650, 79-83.

Ejdrup, T., Lemke, H. T., Haldrup, K., Nielsen, T. N., Arms, D. A., Walko, D. A., Miceli, A., Landahl, E. C., Dufresne, E. M. \& Nielsen, M. M. (2009). J. Synchrotron Rad. 16, 387-390.

Kraft, P., Bergamaschi, A., Brönnimann, C., Dinapoli, R., Eikenberry, E. F., Graafsma, H., Henrich, B., Johnson, I., Kobas, M., Mozzanica, A., Schlepütz, C. M. \& Schmitt, B. (2009). IEEE Trans. Nucl. Sci. 56, 758-764.

Ponchut, C., Visschers, J. L., Fornaini, A., Graafsma, H., Maiorino, M., Mettivier, G. \& Calvet, D. (2002). Nucl. Instrum. Methods Phys. Res. A, 484, 396-406.

Reinhardt, M., Koc, A., Leitenberger, W., Gaal, P. \& Bargheer, M. (2016). J. Synchrotron Rad. 23, 474-479.

Schoenlein, R. W., Chattopadhyay, S., Chong, H. H. W., Glover, T. E., Heimann, P. A., Leemans, W. P., Shank, C. V., Zholents, A. \& Zolotorev, M. (2000). Appl. Phys. B, 71, 1-10.

Wullf, M., Plech, A., Eybert, L., Randler, R., Schotte, F. \& Anfinrud, P. (2003). Faraday Discuss. 122, 13-26. 